\title{
Research on the Space Deployable Mechanism Driven by Twist Spring
}

\author{
Weiguo Zhao ${ }^{1, \text { a }}$, Yanchun $\mathrm{Li}^{1, \mathrm{~b}}$, Yijian Wang ${ }^{1, \mathrm{c}}$, Boqian $\mathrm{Xu}^{1, \mathrm{~d}}$, Yan Wang ${ }^{1, \mathrm{e}}$ \\ ${ }^{1}$ Changchun Institute of Optics, Fine Mechanics and Physics, Changchun 130033, China; \\ az-w-g@163.com, bfeilong99031@126.com, cwangyijian121@126.com, 'ciomp_xubq@126.com
}

Keywords: Deployable mechanism, twist spring driving, dynamic analysis.

\begin{abstract}
A deployable mechanism applied in the aerospace with twist springs and links driving, locking function is described in this paper. Dynamic analysis on this mechanism has been presented, and dynamic simulation has been implemented following the results of analysis, the dynamic characteristics of mechanism have been verified.
\end{abstract}

\section{Introduction}

Because the constraint of limit space in the launch vehicle, large space mechanism usually keep compressed while launching, it's unnecessary to re-compress space mechanism on orbit, and the large one will be deployed all the time. In order to avoid wasting power resource on the satellites or space station, the driving of mechanism usually applies the storage and release of elastic energy rather than using a motor. Due to the high compression ratio, links mechanism with twist springs in joints is always used to implement the deploying of large space mechanism, and it could meet both requirements of compression volume and energy storage. This kind of links mechanism has been applied in many space satellites, such as the full-frame antenna of Russia [1,2].

This paper will introduce and study a type of links mechanism driven by twist spring for large space deployable mechanism. Firstly, the design of this mechanism is introduced, then dynamic analysis and simulation is studied, finally the results of simulation are verified.

\section{Mechanical Design}

A deployable mechanism unit is composed of 4 joints and 3 links between these joints, and the power source is 4 pre-compressed twist springs. 23-links mechanism units are arranged symmetrically to be a pair of unit. One end of the deployable mechanism units is fixed and another end is connected to a moving plate. There is a linear guide mechanism on the plate. Fig. 1 shows the schematic diagram of a pair of 3-links mechanism unit.

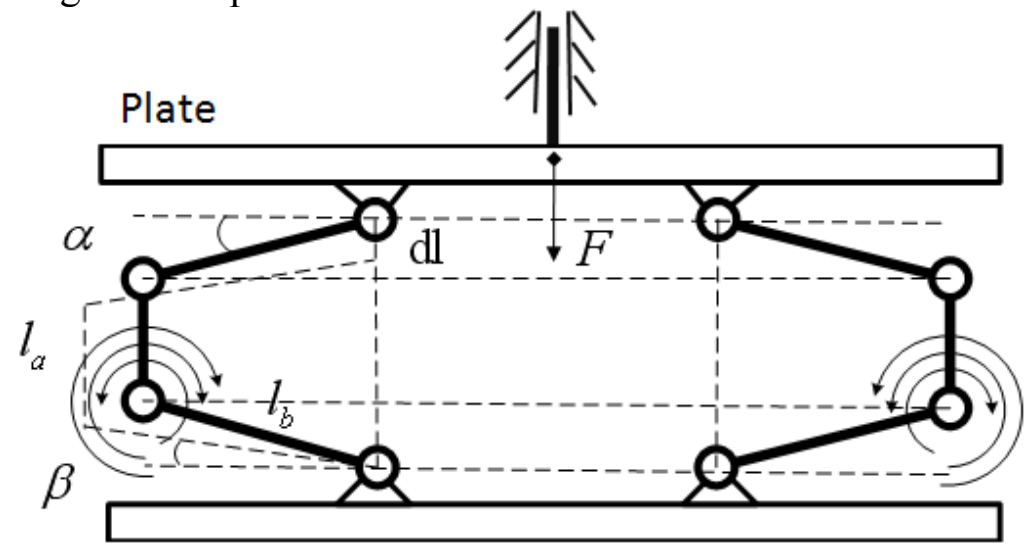

Fig. 1 Schematic diagram of a pair of 3-links deployable mechanism unit

The joint which determines the success of deploy is an important part of mechanism, it is shown in Fig. 2. Twist springs are located around the joint axis in the joints. When mechanism is compressed, the twist springs are deformed and then elastic energy is stored. As deploying, the twist springs release those elastic energy to drive joints, and the mechanism is deployed. To be in the required 
position after deploying, a locking mechanism composed of compression spring and locking pin is needed [3].

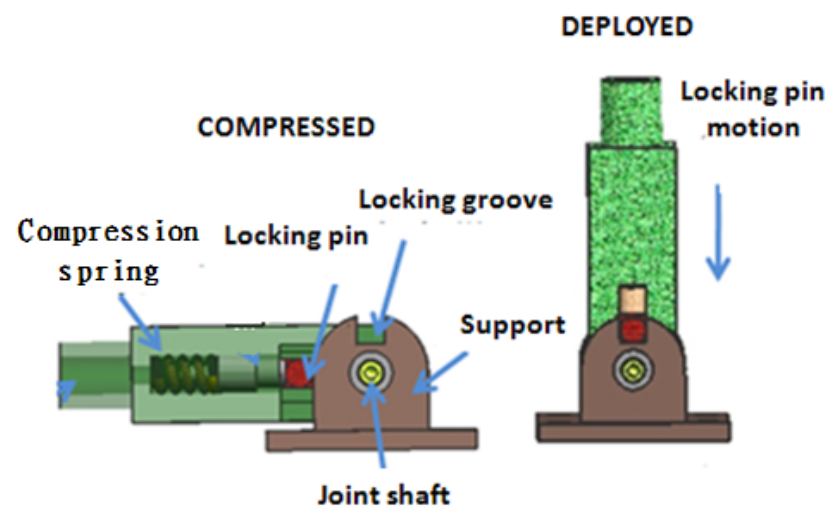

Fig. 2 Compressed and deployed state of a joint

The equipment driven by the deployable mechanism is a large cylinder, so as to provide sufficient elastic power, 4 pairs of deployable mechanism units are symmetrically arranged along the tangential direction.

\section{Dynamic Analysis}

Dynamic equation of deployable mechanism is quite important for mechanical design. Xuesong Qiu has analyzed the similar links mechanism with 2 links according to the conservation of energy [4], the torque of spring is achieving and the stiffness is determined. However, the torque of twist spring is regarded as a constant in the derivation, the connection between the torque and deployed angle is ignored, so the equivalent force of mechanism is also a constant. These are quite different from the actual conditions.

In this paper, the function between the torque and deployed angle of twist spring is imported. The dynamic analysis is implemented according to virtual work principle and the conservation of energy. It is assumed that the position of links is symmetrical and the mechanism is in balance state at any time during deployment, the plate moves a little distance $\mathrm{dl}$ under the force $\mathrm{F}$. The derivation is shown as follows:

According to the geometric relation shown in Fig. 1,

$l=2 l_{b} \sin \alpha+l_{a}$

Where, 1 is the height of links mechanism, $l_{a}$ and $l_{b}$ are the length of links, and a represents the angle that links deploys from compressed state.

The differential relation is given by

$d l=2 l_{b} \cos \alpha \cdot d \alpha$

Twist spring stiffness is $k$, and the maximum work torque is $T_{\max }$, then the spring's torque $T$ is described by

$T=T_{\max }-\mathrm{k} \alpha$.

The friction due to the joint's shaft and locking pin, $\mathrm{T}_{\mathrm{f} 1}$ and $\mathrm{T}_{\mathrm{f} 2}$ are given by

$$
\begin{aligned}
& T_{\mathrm{f} 1}=\mu\left(\frac{F}{2}+\mathrm{m}_{\mathrm{j}} \mathrm{g}\right) \cdot \mathrm{r}_{\mathrm{xz}} . \\
& T_{\mathrm{f} 2}=\mu \cdot \mathrm{k}_{\mathrm{y}} \cdot \Delta_{y 0} \cdot \mathrm{r}_{\mathrm{y}} .
\end{aligned}
$$

The other parameters in these two equations are related to friction calculation.

In this process, the work done by external force and resistance force can be described by

$W_{F}=F \cdot d l$

$W_{\mathrm{j}}=\mathrm{m}_{\mathrm{j}} \mathrm{gdl}$.

$W_{T}=8 \cdot\left(\bar{T}-\mathrm{T}_{\mathrm{f} 1}-\mathrm{T}_{\mathrm{f} 2}\right) \cdot d \alpha$. 
Where $\mathrm{W}_{\mathrm{F}}$ is the work done by external force, $\mathrm{W}_{\mathrm{j}}$ is the change of links' gravity energy, and $\mathrm{W}_{\mathrm{T}}$ is the sum of twist springs' and friction's work.

According to the conservation of energy,

$W_{T}=W_{F}+W_{\mathrm{j}}$.

Another equation will be obtained by substituting above equation into this equation,

$$
\begin{aligned}
& F d l+2 \mathrm{~m}_{\mathrm{j}} \mathrm{g} \frac{d l}{2}=8 \cdot\left(\bar{T}-\mathrm{T}_{\mathrm{f} 1}-\mathrm{T}_{\mathrm{f} 2}\right) \cdot d \alpha \\
& =8 \cdot\left[T_{\max }-k\left(\alpha+\frac{d \alpha}{2}\right)-\mu\left(\frac{F}{2}+\mathrm{m}_{\mathrm{j}} \mathrm{g}\right) \cdot \mathrm{r}_{\mathrm{xz}}-\mu \cdot \mathrm{k}_{\mathrm{y}} \cdot \Delta_{y 0} \cdot \mathrm{r}_{\mathrm{y}}\right] \cdot d \alpha
\end{aligned}
$$

Then,

$$
\begin{aligned}
& F=\frac{-\left(2 k d \alpha+4 k \alpha+m_{j} g l_{b} \cos \alpha\right)+\left(4 T_{\max }-4 \mu m_{j} g r_{x z}-4 \mu k_{y} \Delta_{y 0} r_{y}\right)}{l_{b} \cos \alpha+2 \mu r_{x z}} \\
& =\frac{-\left(2 k d \alpha+4 k \alpha+m_{j} g l_{b} \cos \alpha\right)+C_{1}}{l_{b} \cos \alpha+C_{2}}
\end{aligned}
$$

Assumed that $\mathrm{d} \alpha$ is infinitesimal, this formula can be simplified by

$$
F=\frac{-\left(4 k \alpha+m_{j} g l_{b} \cos \alpha\right)+C_{1}}{l_{b} \cos \alpha+C_{2}}=\frac{C_{3} \alpha+C_{4} \cos \alpha+C_{1}}{C_{5} \cos \alpha+C_{2}}
$$

Where $C_{i}(i=1,2,3,4,5)$ is constant, $F$ is regarded as the equivalent force of a pair of 3-links deployable mechanism unit, further the dynamic equation of total mechanism could be easily obtained according to the Newton law $\mathrm{F}=$ ma. And the function graph of $\mathrm{F}$ is shown in Fig. 3 . Obviously F couldn't be regarded as a constant.

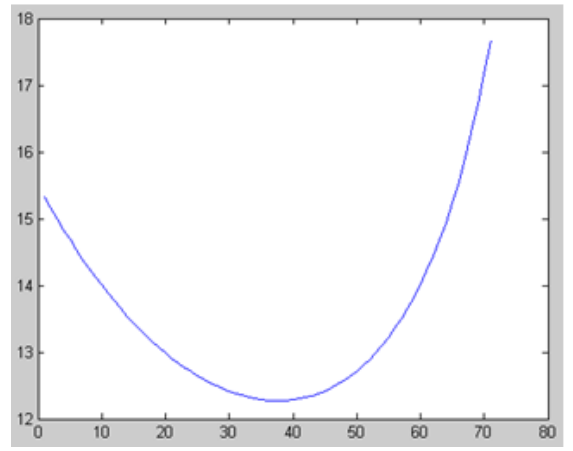

Fig. 3 The graph of $F$ and $a$

Then,

$$
d F=\frac{\left(C_{3}-C_{4} \sin \alpha\right) \cdot d \alpha}{C_{5} \cos \alpha+C_{2}}+\frac{\left(C_{3} \alpha+C_{4} \cos \alpha+C_{1}\right)}{\left(C_{5} \cos \alpha+C_{2}\right)^{2}} \cdot C_{5} \sin \alpha \cdot d \alpha
$$

Finally the equivalent stiffness of a pair of 3-links deployable mechanism unit is given by

$$
K=\frac{d F}{d l}=\frac{C_{3} C_{5} \cos \alpha+C_{3} C_{5} \alpha \sin \alpha+\left(C_{1} C_{5}-C_{2} C_{4}\right) \sin \alpha+C_{2} C_{3}}{2 C_{5} \cos \alpha\left(C_{5} \cos \alpha+C_{2}\right)^{2}}
$$

The equivalent stiffness $\mathrm{K}$ can be used to calculate the multi-floors deployable mechanism. In the simplified model, the mechanism is regarded as a compression spring, and the multi- floors system is composed of spring and mass as shown in Fig. 4. Four pairs of mechanism units are arranged in parallel for each floor, then the total stiffness changes to $4 \mathrm{~K}$.

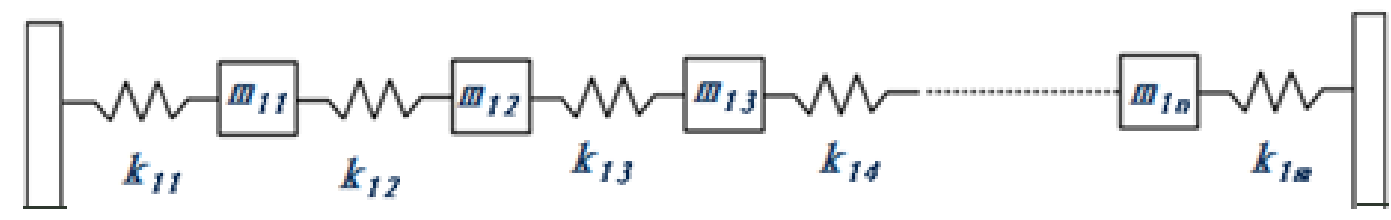

Fig. 4 The multi-floors $\mathrm{K}$ and mass model 


\section{Dynamic Simulation}

From the above analysis, the twist spring stiffness and twist spring parameters can be determined, then dynamic simulation will be carried out.

The dynamic simulation is implemented in Siemens UG NX8.0 simulation module, the stiffness of twist spring in the joint is $1.5 \mathrm{~N} \cdot \mathrm{mm} / \mathrm{deg}$, there is additional rotation damping $10 \mathrm{~N} \cdot \mathrm{mm} \cdot \mathrm{sec} / \mathrm{deg}$ in each joint for simulate the actual friction, the spring stiffness of locking mechanism is $2 \mathrm{~N} / \mathrm{mm}$. And there is also a 3D contact setting for the locking pin, some revolute joints and shifting pair are also added to the joints and plate. The dynamic graph of plate included displacement, velocity and acceleration are shown in Fig.5. These dynamic characteristics could meet the functional requirements of customers.

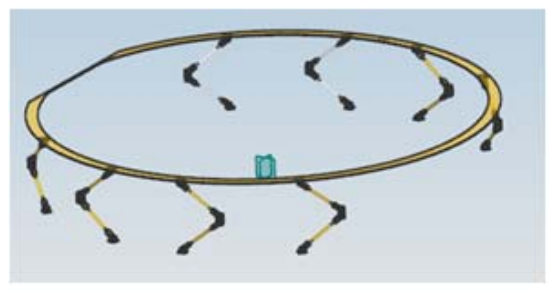

a) Simulating process

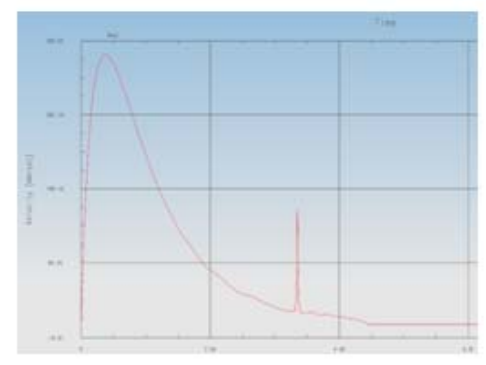

c) Velocity-time curve

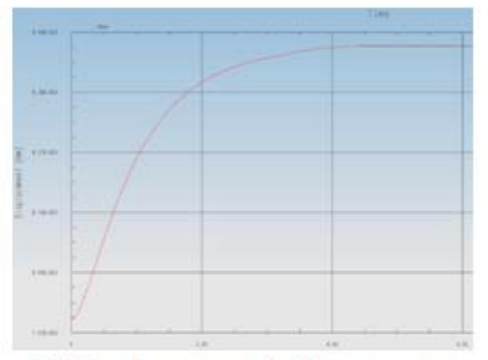

b) Displacement-time curve

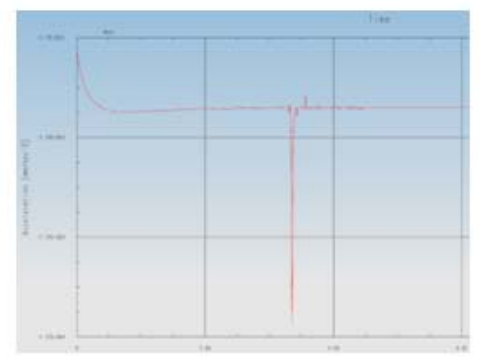

d) Acceleration-time curve

Fig. 5 Displacement, velocity and acceleration graph of deployable mechanism

\section{Conclusions}

In the paper, a deployable space mechanism composed of 3-links mechanism and twist springs driving is proposed, the dynamic model is also established, functions of equivalent force, equivalent stiffness and spring stiffness are obtained, finally dynamic simulation based on the analysis results is implemented, the dynamic characteristics are verified.

\section{References}

[1] Miura.K, Furuya.H. Variable Geometry Truss and Its Application to Deployable Truss and Space Crane. Acta.Astronautics. Vol. 12 (1985) No. 7-8, p. 599-607.

[2] Wujun Chen, Shilin Dong, Gongyi Fu, et al. Structural Analysis for Deployable Space Truss with Twist Springs Activated. Journal of Shanghai Jiaotong University. Vol. 34 (2000) No.8, p. 1074-1077.

[3] Tiehua Xie, Fuling Guan, Bin Su, et al. Research and Analysis on Dynamic Properties of Spatial Rod-cable Deployable Structure. Spatial Structures. Vol. 3 (2004) No.3, p. 48-54.

[4] Xuesong Qiu, Zongquan Deng, Ming Hu, et al. Design and study on space spring deployable mechanism. Journal of Machine Design. Vol. 22 (2005) No. 10, p. 25-27. 